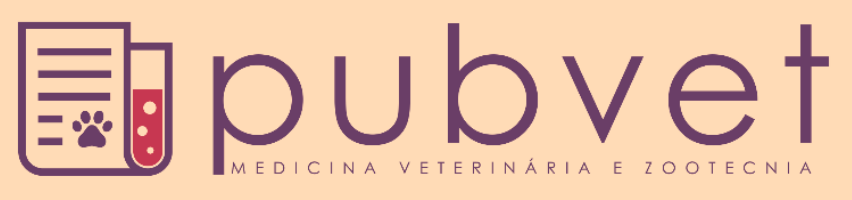

https://doi.org/10.31533/pubvet.v16n01a1024.1-16

\title{
Dinâmica do bem-estar de bovinos leiteiros acometidos por podopatias como suporte para a perícia veterinária
}

\author{
Jackson Barros do Amaral ${ }^{1}$, Luís Alberto Ambrósio ${ }^{1}$ (๑)

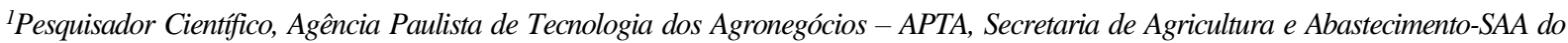 \\ Estado de São Paulo, Instituto de Zootecnia, Centro de Pesquisa de Bovinos Leite, Rua Heitor Penteado, 56, Nova Odessa, SP, Brasil, Cep \\ 13.380-011 *Autor para correspondência, e-mail:jackson5amaral@gmail.com,jackson.amaral@sp.gov.br.
}

Resumo. A perícia de bem-estar de bovino leiteiro é uma demanda inovadora e relevante na investigação legal e forense. Em particular, devido à necessidade de tratamento e prevenção das doenças da produção, entre elas as podopatias. Os pareceres técnicos veterinários podem constatar a negligência, imperícia e imprudência dos responsáveis pelos cuidados da saúde animal e subsidiar a tomada de decisão judicial, quando cabível, em casos de maus-tratos, crueldade e abuso em bovinos. O entendimento da complexidade das podopatias causadas por múltiplos fatores com interações não lineares com o bem-estar requer abordagem dinâmica. A metodologia de Dinâmica de Sistemas foi usada para construir o modelo conceitual dos problemas das podopatias e das relações de causa e efeito dos construtos baseados nos conhecimentos e experiências dos pesquisadores e na literatura. $\mathrm{O}$ modelo contém seis diagramas com ciclos de reforço e de balanço e os respectivos padrões de referências das variáveis estados. As hipóteses dinâmicas realçaram o papel da perícia veterinária ao considerar a inter-relação de reforço entre podopatias e bem-estar que direciona o sistema para círculos virtuosos ou viciosos. Foi detalhado como as podopatias causam dor e sofrimento reforçando ainda mais a perda de bem-estar. A ação do médico veterinário, motivada por questões éticas, legais ou sociais, ao promover a prevenção e tratamento gera retroalimentações de balanço que podem elevar o bem-estar para o nível desejado. Os ciclos de balanço da prevenção possuem demoras nos efeitos das ações sobre a incidência de podopatias, com consequente continuidade, durante algum tempo, da dor e sofrimento dos animais. Do modelo emergiu a sinergia contraintuitiva entre aplicação de recursos financeiros para a produção e investimentos para assegurar o bem-estar. Assim, promover os ciclos de balanço da saúde, diminuindo as podopatias e melhorando o bem-estar animal, influenciará positivamente os ciclos de reforço da economia. A perícia por meio de retroalimentação negativa baseada nos valores técnicos, legais, éticos e humanitários controla as podopatias e influencia na adoção de ações de melhoria do bem-estar. Concluiu-se que o modelo conceitual é um framework para auxiliar a modelagem computacional e contribui para a compreensão da complexidade das podopatias em questões legais e forenses. Ações humanas influenciam a dinâmica das podopatias comprometendo o bem-estar podendo gerar provas de maus-tratos em bovinos leiteiros.

Palavras-chave: Afecções podais, bovino, medicina veterinária legal, modelo conceitual, retroalimentação

\section{Dynamics of the welfare of dairy cattle affected by podopathies as support for veterinary expertise}

Abstract. Due to the necessity for treatment and prevention of production diseases, such as podopathies, dairy cattle welfare expertise is an innovative and relevant demand in legal and forensic investigations. Veterinary technicians can establish the negligence, malpractice, and imprudence in animal care, and would support judicial decision, in cases 
of cattle mistreatment, cruelty, and abuse. A dynamic approach is required to understand the podopathies complexity due to non-linear interactions between multifactors and cattle welfare. System Dynamics methodology was used to build a conceptual model of podopathies problems and constructs cause-and-effect relationships based on knowledge and experience of the researchers and literature. The model contains six diagrams with reinforcing and balancing cycles and the respective state variables reference patterns. The dynamic hypotheses highlighted the role of veterinary expertise in assessing the reinforcing the podopathies and welfare interaction that drives the system into a virtuous or vicious circle. It was detailed how podopathies cause pain and suffering, further reinforcing the loss of welfare. The veterinarian's action, motivated by ethical, legal, or social pressure, to promote prevention and treatment, creates balance feedbacks that might elevate welfare to the desired level. The prevention cycles have delayed actions effects on podopathies, resulting in the continuity of pain and suffering, for some time. A counterintuitive synergy emerged between the use of financial resources for production and investments to ensure welfare. As a result, promoting health balancing cycles, reducing podopathies and improving animal welfare positively influence economic. Expertise through negative feedback based on technical, legal, ethical, and human values controls the podopathies incidence influencing actions to improve the welfare. It was concluded that the conceptual model serves as a framework to aid computational modeling and contributes to better understanding of the podopathies complexity in legal and forensic matters. Human actions influence podopathies dynamics, which compromise animal welfare and may lead to evidence of abuse in dairy cattle.

Keywords: Foot disorders, bovine, forensic veterinary medicine, conceptual model, feedback

\section{Dinámica del bienestar del ganado lechero afectado por podopatías como apoyo a la pericia veterinaria}

Resumen. Debido a la necesidad de tratamiento y prevención de enfermedades de la producción, como las podopatías, la pericia en el bienestar del ganado es una demanda innovadora y relevante en las investigaciones legales y forenses. Los técnicos veterinarios pueden establecer la negligencia, impericia y la imprudencia en el cuidado de los animales, y apoyarían la decisión judicial, en casos de maltrato, crueldad y abuso del ganado. Se requiere un enfoque dinámico para comprender la complejidad de las podopatías debido a interacciones no lineales entre multifactores y el bienestar del ganado. Se utilizó la metodología de la Dinámica de Sistemas para construir un modelo conceptual de problemas de podopatías y construir relaciones de causa y efecto basadas en el conocimiento y experiencia de los investigadores y la literatura. El modelo contiene seis diagramas con ciclos de refuerzo y balance y los respectivos patrones de referencia de las variables de estado. Las hipótesis dinámicas destacaron el papel de la pericia veterinaria en la evaluación del refuerzo de las podopatías y la interacción del bienestar que lleva al sistema a un círculo virtuoso o vicioso. Se detalló cómo las podopatías provocan dolor y sufrimiento, reforzando aún más la pérdida de bienestar. La acción del veterinario, motivada por la presión ética, legal o social, para promover la prevención y el tratamiento, crea retroalimentaciones de balance que pueden elevar el bienestar al nivel deseado. Los ciclos de prevención tienen efectos de acción retardada en las podopatías, resultando en continuidad del dolor y el sufrimiento, durante algún tiempo. Surgió una sinergia contra intuitiva entre el uso de recursos financieros para la producción y las inversiones para garantizar el bienestar. Como resultado, la promoción de los ciclos de balance de la salud, la reducción de las podopatías y mejor bienestar animal influyen positivamente en la economía. La pericia a través de la retroalimentación negativa basada en valores técnicos, legales, éticos y humanos controla la incidencia de las podopatías influyendo en las acciones para mejorar el bienestar. Se concluye que el modelo conceptual sirve de marco para ayudar la modelización computacional y contribuye a una mejor comprensión de la complejidad de las podopatías en materia jurídica y forense. Las acciones humanas influyen en la dinámica de las podopatías, que comprometen el bienestar animal y pueden dar lugar a pruebas de maltrato en el ganado lechero. 
Palabras clave: Trastornos del pie, bovino, medicina veterinaria forense, modelo conceptual, retroalimentación

\section{Introdução}

O termo "doenças da produção" (Payne, 1972) é conceituado como "doenças causadas pelo homem" as quais vêm aumentando com a pressão de alta produtividade na pecuária intensiva (Hemsworth et al., 1995). Entre estas, as podopatias afetam os bovinos leiteiros e resultam em problemas de saúde, claudicação e dor (Bruijnis et al., 2012; Silva et al., 2015). O bem-estar animal vem aprimorando o senso crítico da necessidade de tratamento e prevenção das doenças da produção. O consumidor está atento para os produtos oriundos de boas práticas de criação e preservação ambiental (Braga et al., 2018; Queiroz et al., 2014). A preocupação com o bem-estar e controle da dor e do sofrimento é importante por agregar valor ao produto e viabilizar financeiramente o agronegócio (Katzenberger et al., 2020; Luna, 2008).

O controle da dor ainda é negligenciado, apesar da crescente preocupação nacional e internacional com o bem-estar animal. $\mathrm{O}$ uso de técnicas arcaicas às vezes não é percebido ou é ocultado pelos proprietários e reveladas apenas nos exames de rotina (Amaral et al., 2018). O exame clínico é importante na garantia do bem-estar de bovinos esclarece o diagnóstico, tratamento e prognóstico das enfermidades (Feitosa, 2014). A perícia veterinária envolve conhecimentos técnico-científicos para esclarecer vestígios e interpretações de fatos perante acontecimentos ilícitos ou suspeitos envolvendo animais (Tremori \& Rocha, 2013).

As podopatias são dinâmicas, evoluem ao longo do tempo, como comprovam estudos clínicos de tempo de recuperação com o tratamento e recorrência de animais afetados (Berry et al., 2012; Yang et al., 2020). A perícia veterinária deve considerar o tempo como uma variável nas avaliações do bemestar e suas relações com a dor e sofrimento de animais.

O objetivo é apresentar as relações dinâmicas de causa e efeito entre podopatias, dor, sofrimento e bem-esta e discutir suas implicações técnicas e legais aplicadas à perícia veterinária.

\section{Material e métodos}

\section{Base de informações}

O modelo conceitual, no contexto da bovinocultura leiteira foi construído com base nos conhecimentos e experiências dos pesquisadores e nas informações da literatura sobre as relações entre as podopatias, dor, sofrimento e bem-estar e suas implicações técnicas e legais. Os principais construtos do modelo conceitual são apresentados no Quadro 1.

Quadro 1. Descrições dos construtos principais do modelo conceitual de podopatias em bovinos leiteiros.

\begin{tabular}{|l|l|}
\hline Construtos & \multicolumn{1}{c|}{ Descrição } \\
\hline Podopatias & $\begin{array}{l}\text { Em bovinos, são patologias podais que apresentam causas multifatoriais envolvendo fatores de ordem } \\
\text { infecciosa, traumática ou ambiental (Amaral \& Trevisan, 2017; Ollhoff \& Ortolani, 2001; Silva et al. } \\
\text { 2016). }\end{array}$ \\
\hline Dor & $\begin{array}{l}\text { Dor é uma experiência sensitiva e emocional desagradável, associada a uma lesão tecidual real ou } \\
\text { potencial (IASP, 2020). }\end{array}$ \\
\hline Sofrimento & $\begin{array}{l}\text { Sofrimento é um estado emocional negativo que deriva de circunstâncias físicas, fisiológicas e } \\
\text { psicológicas adversas de acordo com as capacidades cognitivas da espécie e do ser individual e com sua } \\
\text { experiência de vida (Morton \& Hau, 2002). }\end{array}$ \\
\hline Bem-estar & $\begin{array}{l}\text { Bem-estar de um indivíduo é o seu estado em relação às suas tentativas de adaptar-se ao seu ambiente } \\
\text { Broom, 1986). }\end{array}$ \\
\hline Tratamento & $\begin{array}{l}\text { É o conjunto de meios biológicos, cirúrgicos, farmacológicos, físicos, higiênicos e psíquicos utilizados } \\
\text { para curar, atenuar ou abreviar uma doença (Infopédia, 2021). }\end{array}$ \\
\hline Maus-tratos & $\begin{array}{l}\text { Qualquer ato direto ou indireto, comissivo ou omissivo, que intencionalmente ou por negligência, } \\
\text { imperícia ou imprudência provoque dor ou sofrimento desnecessário aos animais (Brasil, 2018). }\end{array}$ \\
\hline Prevenção & $\begin{array}{l}\text { Prevenção das podopatias incluem todos os fatores que contribuem para instalação, manutenção e } \\
\text { disseminação destas afecções podais, e seus mecanismos de transmissão e medidas utilizadas para seu } \\
\text { controle (Bond et al., 2012; de Vries et al., 2013). }\end{array}$ \\
\hline Fatores de risco & $\begin{array}{l}\text { Em podopatias incluem todos aqueles fatores que contribuem direta ou indiretamente na ocorrência de } \\
\text { afecções podais (Braga et al., 2018; de Vries et al., 2013). }\end{array}$ \\
\hline
\end{tabular}




\section{Metodologia de dinâmica de sistemas}

A Dinâmica de Sistema lida com o modo de como a estrutura de um sistema e seus fluxos de informação determinam o comportamento do sistema: o controle do crescimento, a estabilidade, o declínio, o sucesso e o fracasso. Seu campo de atuação concentra-se no modo como as relações entre ciclos de retroalimentação internos causam a mudança de um sistema ao longo do tempo. Compreender por que um sistema tem um determinado comportamento permite redesenhar sua estrutura e adotar políticas para melhorar o seu comportamento. As ideias e os métodos de Dinâmica de Sistemas são aplicáveis aos sistemas naturais, humanos e tecnológicos. Os estudos com base na Dinâmica de Sistemas combinam teoria e simulação computacional com aplicação muito prática para os problemas do mundo real (Forrester, 1971).

Aplicou-se a metodologia de Dinâmica de Sistema (Senge, 2005; Sterman, 2000) para construir modelos conceituais que tornam explícitas as relações complexas de causa e efeito, não lineares, entre as podopatias, a dor, o sofrimento e o bem-estar animal da bovinocultura leiteira. Esta metodologia vem sendo usada na etologia aplicada para avaliar a complexidade das relações homem-animal integrando o conhecimento multidisciplinar em busca de soluções práticas para problemas subjacentes (Costa et al., 2021; Toledo et al., 2018). O uso da Dinâmica de Sistemas na prática dos médicos veterinários é encorajado por Groves (2018) e vem sendo aplicada em várias áreas da pesquisa veterinária (Voyer \& Jordan, 2018; Wilson et al., 2017).

A Dinâmica de Sistemas é focada nas retroalimentações positivas e negativas entre os componentes do sistema associadas aos problemas emergentes de origens endógenas. O modelo conceitual é apresentado em um diagrama de ciclo causal (CLD - Causal Loop Diagram). OCLD é uma ferramenta gráfica para comunicar as hipóteses dinâmicas sobre as relações entre as variáveis relacionadas ao problema estudado. O Modelo computacional baseado na estrutura do modelo conceitual simula cenário gerando as curvas de referência BOT (Behavior Over Time) que mimicam as trajetórias das variáveis estado do sistema conforme estabelecido nos arquétipos sistêmicos.

Com base nas evidências da literatura foram definidas as hipóteses dinâmicas relacionadas às relações de causa e efeito entre os construtos presentes no modelo conceitual. Essas hipóteses foram representadas nos CLD e nos gráficos BOT e caracterizadas pela inter-relação dos ciclos de retroalimentação de Reforço (R), indicando que as variáveis do sistema apresentam um crescimento exponencial ou uma tendência de declínio acelerado ao longo do tempo e dos ciclos de retroalimentação de Balanço (B) indicando que as variáveis tendem a um patamar de equilíbrio, desejado ou não desejado, resultando em círculos virtuosos ou viciosos (Senge et al., 2012).

\section{Modelos conceituais e diagramas de ciclos causais}

O Modelo conceitual se constitui no framework referencial para a construção do modelo computacional, o qual produzirá as informações quantitativas da dinâmica do sistema a serem usadas nas decisões e formulação de políticas ou ações. O CLD apresenta as estruturas das retroalimentações e das demoras (delays) entre os efeitos das variáveis a partir das informações básicas de polaridade das suas influências causais (Sterman, 2000; Senge et al., 2012). No CLD, as setas de influências indicam as direções das relações de causa e efeito. Assim, se um evento ocorrer na variável causadora (localizada no início da seta) provocará um efeito específico na variável afetada (localizada na ponta da seta), Figura 1. A polaridade da influência de uma variável sobre a outra é entendida como relação de causalidade. A polaridade das relações causais é condição necessária para converter o conhecimento sobre a estrutura em conhecimento sobre o comportamento do sistema (Richardson, 1995).
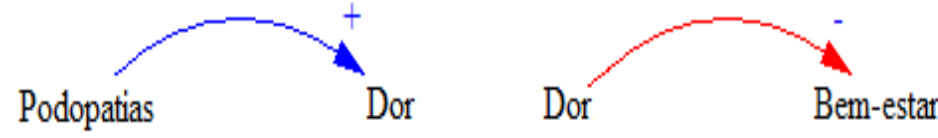

\section{Influência com polaridade positiva $(+) \quad$ Influência com polaridade negativa $(-)$}

Figura 1. Polaridade positiva e negativa da influência de uma variável sobre outra. Na relação de polaridade positiva (+), a influência ocorre na mesma direção, por exemplo, um aumento na ocorrência de podopatias aumenta a dor dos animais ou a diminuição das podopatias diminuirá a dor. Na relação de polaridade negativa (-) a influência ocorre em direção oposta, por exemplo, o aumento da dor dos animais diminuirá o bem-estar ou a diminuição da dor aumentará o bem-estar. 
Um ciclo de retroalimentação causal é representado por um círculo fechado formado por variáveis interligadas por setas de influências. A combinação de influências com polaridades positivas e negativas geram dois tipos de ciclos de retroalimentação: Ciclo de Reforço (R) e Ciclo de Balanço (B), Figura 2. A polaridade dos ciclos de retroalimentação é definida em função da quantidade de influências com polaridade negativa. Quando a quantidade de influências negativas for nula ou par, a polaridade do ciclo de retroalimentação será positiva e o ciclo será de Reforço. Quando a quantidade de influências negativas for ímpar, a polaridade do ciclo de retroalimentação será negativa e o ciclo será de Balanço. No Ciclo de Reforço, as interações são tais que cada ação no sistema contribui mais para a mesma ação, produzindo um crescimento exponencial ou colapso nas variáveis. No Ciclo de Balanço, as influências de compensação atuam para levar as variáveis (dependentes e independentes) para uma concordância de comportamento ou a uma estabilidade (homeostase) gerando uma curva com patamar máximo ou mínimo. Assim, com base em Bicalho et al. (2009) há uma retroalimentação positiva (R) entre claudicação e a dor do animal com lesão podal, onde o aumento da dor tem como resposta o aumento da claudicação e o aumento da claudicação associada à debilidade do animal contribui para aumentar a dor. Na avaliação de laminite, a postura de claudicação afeta a lesão podal e a lesão afeta a postura, não podendo se estabelecer uma relação de causa e efeito em via única (Capion et al., 2008). A retroalimentação negativa (B) entre o tratamento e as podopatias leva ao controle da doença, onde o aumento do tratamento diminui as podopatias e o aumento no reconhecimento e diagnóstico das podopatias levam ao aumento do seu controle por meio de mais tratamento (Silva et al., 2006), ao longo do tempo, até obter um resultado efetivo, conforme Figura 2.
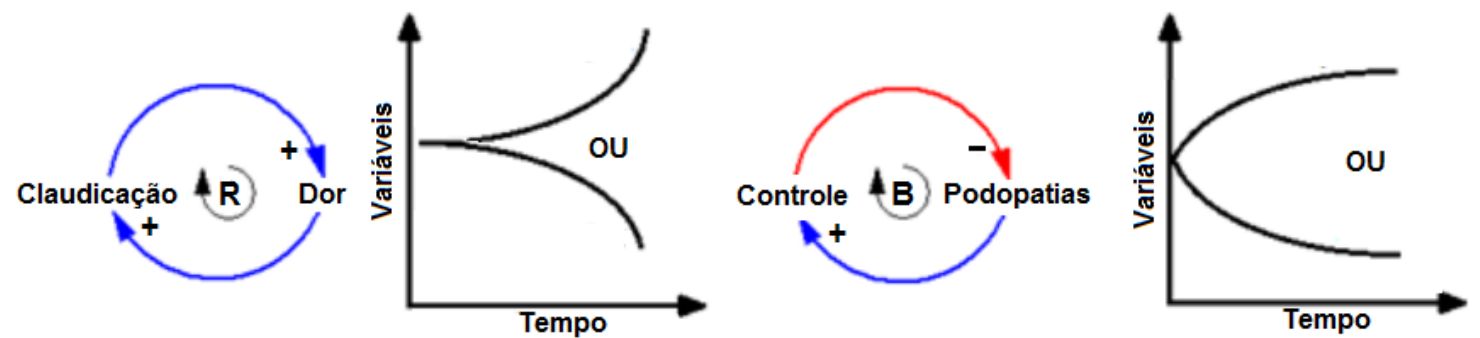

Figura 2. Polaridade dos ciclos de retroalimentação, Ciclo de Reforço (R) e Ciclo de Balanço (B). Os gráficos BOT ilustram as hipóteses dinâmicas para os padrões de referência conceitual do comportamento de cada ciclo. O software Vensim PLE ${ }^{\circledR}$ para Windows, Versão 8.1.1 (Ventana Systems, Inc., Harvard, MA) foi usado para construir os modelos conceituais.

\section{Resultados e discussão}

\section{Descrição narrativa do modelo conceitual}

No sistema podopatias-bem-estar animal estão presentes problemas recorrentes de dor e sofrimento dos animais acometidos por podopatias que comprometem o bem-estar dos animais na bovinocultura leiteira que está inserida no sistema social maior. A dinâmica dos sistemas de produção de leite conduzidos sob a ótica econômica estrita produz comportamentos inesperados, ineficazes ou resultados prejudiciais frequentemente rejeitados pela sociedade. A legislação de bem-estar e proteção animal vem sendo debatida e promovendo impacto positivo, mas ainda se mostram ineficazes quando se trata de animais de produção. Seus resultados costumam ficar aquém das expectativas. Conforme Forrester (1992), isto ocorre porque o comportamento dinâmico dos sistemas sociais não é compreendido e as práticas associadas causam exatamente o reverso dos resultados desejados. Na Dinâmica de Sistemas, os modelos conceituais tornam explícitas as relações complexas sistêmicas contribuindo para o entendimento de comportamentos do sistema que são contraintuitivos. De acordo com Amaral \& Trevisan (2017) do ponto de vista médico a dor e o sofrimento são entidades nosológicas complexas cujo diagnóstico, controle e tratamento envolvem interações complexas entre fatores intrínsecos do animal, homem, ambiente e microrganismos como componentes do sistema de produção de bovinos leiteiros.

\section{Dinâmica dos estados do Bem-estar animal e da ocorrência de Podopatias com ciclo de retroalimentação de reforço.}

O comportamento dinâmico das podopatias em rebanhos de bovinos leiteiros, Figura 3 , é direcionado, ao longo do tempo, pelas interações entre os múltiplos fatores de risco de ordem 
infecciosas, traumáticas ou ambientais (Silva et al., 2016; Silva et al., 2006) e fatores genéticos (Nicoletti, 2004). Os efeitos dinâmicos da prevalência das podopatias comprometendo o bem-estar animal são reportados na literatura (Bruijnis et al., 2012).
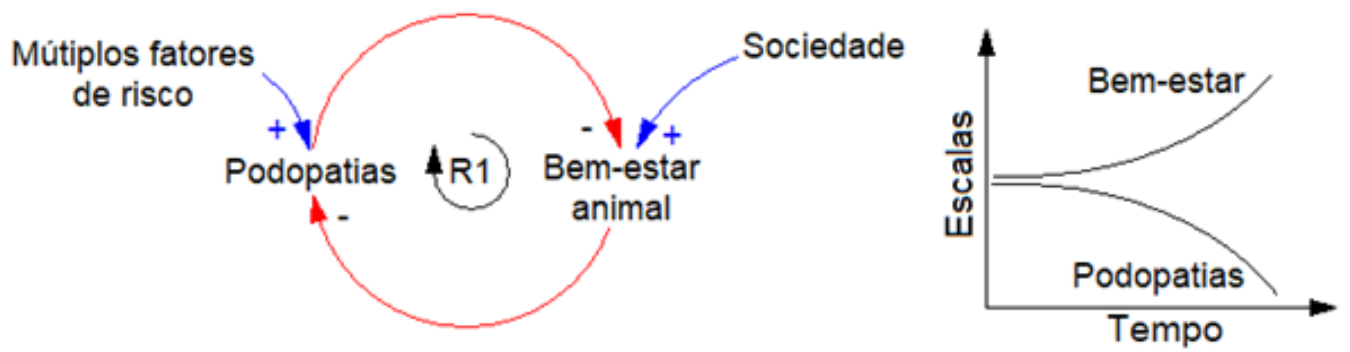

Figura 3. Diagrama de ciclo causal de Podopatias e Bem-estar animal e gráfico BOT de referência. No ciclo de retroalimentação de reforço (R1) o aumento nos eventos associados aos múltiplos fatores de risco resultam no aumento da ocorrência de podopatias em bovinos leiteiros ao longo do tempo.

A influência com polaridade negativa (-) indica que um aumento nas ocorrências de podopatias causarão efeitos em direção oposta, diminuindo o estado de bem-estar animal e eventos que contribuem para o aumento do bem-estar animal influenciam na diminuição da ocorrência de podopatias. O ciclo R1 entre Podopatias e bem-estar animal torna-se um círculo virtuoso dinâmico quando predominam as influências que promovem o bem-estar animal simultaneamente com decrescimento da ocorrência de podopatias e torna-se um círculo vicioso, quando o crescimento da ocorrência de podopatias reforça a diminuição do bem-estar animal. Entretanto, a retroalimentação negativa do bem-estar animal sobre a prevalência e incidência das podopatias é menos intuitiva e pouco pesquisada. Para von Keyserlingk et al. (2009) sob a perspectiva do bem-estar animal grande parte da preocupação com as doenças surge da consequência sobre o sofrimento dos animais afetados. $\mathrm{O}$ bem-estar animal é uma preocupação crescente dos consumidores que buscam informações sobre a qualidade e o modo que seus alimentos são produzidos (Pinto et al., 2020; Weary \& Von Keyserlingk, 2017), também é crescente a percepção dos veterinários para a proteção do bem-estar de bovinos leiteiros (Van Dyke et al., 2021). Além disso, o bem-estar é o foco dos crescentes movimentos pró legalização dos direitos dos animais fundamentados na ética que buscam fazer valer o direito fundamental à existência digna de cada animal (Ataíde Júnior, 2018). Assim, ao perceber a perda de bem-estar animal nos sistemas de produção, os agentes da sociedade exercem pressão que motiva a prevenção, controle e tratamento das podopatias e suas consequências sobre a dor e sofrimento dos animais, conforme representado neste CLD.

\section{Dinâmica dos estados da dor e sofrimento com ciclos de retroalimentação de reforço das podopatias e bem-estar animal}

A dor e sofrimento estão presentes em todos os sistemas de exploração de bovinos leiteiros e as podopatias representam um dos mais importantes problemas de saúde que compromete o bem-estar desta categoria animal (Amaral \& Trevisan, 2017). O impacto da dor causada pelas podopatias no bemestar animal é reportado na literatura como um dos principais problemas na criação de bovinos leiteiros e causam preocupações para produtores e sociedade (Gleerup et al., 2015; McLennan, 2018; Ramanoon et al., 2018). A sociedade vem se tornando sensível a estes problemas, demonstrando sentimentos que não se limitam apenas à crueldade, sendo objetos de pesquisas nacionais e internacionais (Amaral \& Trevisan, 2017). A dor é uma condição dinâmica, o que significa que se não tratada pode aumentar em magnitude e pode levar a condições crônicas que alteram o comportamento de bovinos leiteiros (Gleerup et al., 2017). O CLD da figura 4 torna explícitas as relações complexas sistêmicas nas podopatias que comprometem o bem-estar animal pela evidência de dor e sofrimento.

A evidência de dor e sofrimento dos animais acometidos por podopatias indicam um problema grave de bem-estar dos bovinos (Amaral \& Trevisan, 2017; Bond et al., 2012; Gaynor \& Muir, 2009). Nos ciclos de retroalimentação a dor e sofrimento interagem com a dinâmica do estado de bem-estar formando círculos viciosos ou virtuosos. As setas de influência com polaridade negativa indicam que o aumento da dor e sofrimento causa diminuição do bem-estar animal. O gráfico BOT (Figura 4) apresenta a dinâmica de um círculo vicioso. As podopatias causam lesões que influenciam diretamente a dor e 
sofrimento com polaridade positiva e as podopatias, dor e sofrimento em conjunto influenciam diretamente o estado de bem-estar animal com polaridade negativa formando os ciclos de reforço (R1, R2 e R4) uma vez que a perda de bem-estar, devido à influência negativa provocar aumento das podopatias. $\mathrm{O}$ aumento da dor influencia positivamente no aumento do sofrimento resultando no ciclo de reforço R3. A dor causa sofrimento (Dawkins, 2017; Flecknell, 2008) e o aumento ou intensificação das lesões podais influenciam, na mesma direção, o aumento da dor e do sofrimento dos animais na bovinocultura leiteira (Neveux et al., 2006; Olmos et al., 2009).
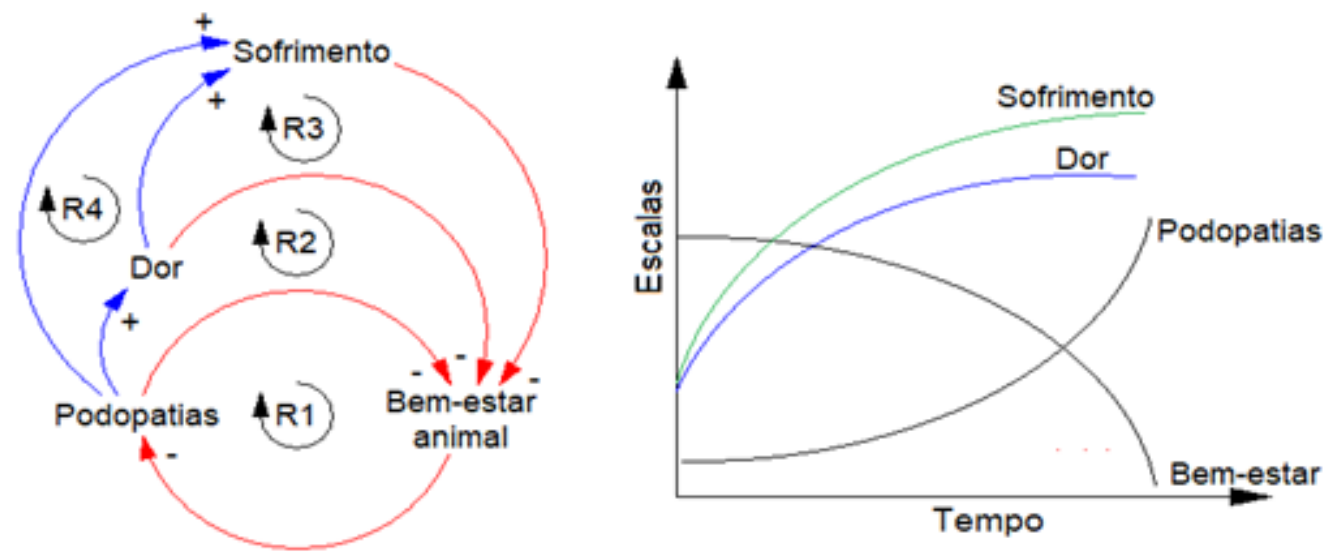

Figura 4. Diagrama de ciclos causais mostrando a hipótese dinâmica das relações de causas e efeitos entre podopatias, dor, sofrimento e bem-estar animal e gráfico BOT de referência do estado do sistema ao longo do tempo. Os ciclos de retroalimentação de reforço (R2, R3 e R4) indicam como a Dor e o Sofrimento atuam reforçando os efeitos da ocorrência das podopatias na diminuição no bem-estar animal.

O papel fundamental para o médico veterinário na sociedade, conforme determinam à ética, o humanitarismo e a legislação, está relacionado direta ou indiretamente às ações de controle da dor e do sofrimento no uso de animais, fazendo valer o bem-estar animal (Brasil, 2016; Brasil, 2018; Amaral \& Trevisan, 2017; Luna, 2008). As interações que ocorrem entre as variáveis nos ciclos de reforço indicam círculos viciosos do sistema quando se aumentam os efeitos dos múltiplos fatores de risco sobre a ocorrência das podopatias e se diminuem as pressões sociais e suas considerações de ética sobre o bemestar animal. Por outro lado, se houver altas pressões sociais e considerações de bem-estaristas e baixos níveis dos fatores de risco, obtém-se um círculo virtuoso do sistema. Entretanto, este sistema é regulado por inúmeros ciclos de Balanço (B) que serão discutidos em seguida, também chamados de ciclos de Equilíbrio, Controle ou Compensadores, os quais direcionam o estado do sistema, em termos de ocorrência das Podopatias e do grau do bem-estar animal, em trajetórias que tendem, ao longo do tempo, para um patamar desejado ou indesejado para variáveis de dor e sofrimento. Assim, torna-se necessário que produtores, veterinários e profissionais que atuam na perícia veterinária entendam tanto da dinâmica das podopatias para promover o bem-estar animal quanto entendam como a dinâmica do bem-estar retroalimenta o sistema por meio do controle da dor e do sofrimento dos animais.

A complexidade da dor ultrapassa a fronteira física e é influenciada pelo meio ambiente e pela resposta psíquica do animal. A dor é considerada como fenômeno biopsicossocial que envolve aspectos biológicos, psíquicos e sociais do indivíduo e, nos animais, sua avaliação é considerada o ponto crítico. Os animais de produção são os que mais sofrem dor, pois raramente recebem profilaxia ou tratamento analgésico, havendo negligência, assim, são submetidos a diversos procedimentos cruentos com finalidade para a produção (Canozzi et al., 2020; Lorena et al., 2013; Luna, 2008). Protocolos que incluem indicadores de comportamento relacionados à dor e sofrimento, como arqueamento do dorso e claudicação associados às podopatias e comportamentos em resposta aos procedimentos de descorna e castração, são importantes para indicar condições clínicas de forma suficientemente precisa para serem utilizados no campo (Amaral et al., 2018; Hammerschimidt \& Molento, 2017; Ting et al., 2003). De fato, a frequência de afecções podais em bovinos é mais alta em sistemas de confinamentos e semiconfinamento do que em animais criados extensivamente (Ollhoff \& Ortolani, 2001). Além disto, bovinos criados em confinamento que conseguem manter um balanço adequado entre crescimento e desgaste do estojo córneo são menos sujeitos aos problemas de cascos (Manson \& Leaver, 1988). 
Dinâmica dos estados da dor e sofrimento com dominância de ciclos de balanço das podopatias e bem-estar animal devido à ação das variáveis tratamento e prevenção.

Os ciclos de retroalimentação de balanço (B), também chamados de ciclos de controle e retroalimentação negativa, contribuem para interpretar e entender o equilíbrio dinâmico dos estados das podopatias, dor e sofrimento e suas influências sobre o bem-estar dos bovinos leiteiros, quando influenciados pelo tratamento e prevenção ao longo do tempo. Os ciclos de balanço também representam processos homeostáticos naturais de autorregulação da dor. Os procedimentos associados ao tratamento das podopatias e da dor incluem diversas fases dependentes de múltiplos fatores inter-relacionados.

Tratar os animais afetados por podopatias não diminui a ocorrência destas afecções, para isso ocorrer, é necessário diminuir os fatores de riscos. Entre os principais fatores de riscos que contribuem para diminuir as ocorrências das podopatias destacam-se as correções das técnicas de manejo, adequação da nutrição, melhoria da higiene do ambiente de criação e adequação das instalações, promover a podologia preventiva dos cascos, utilização de pedilúvios e oferecer conforto aos animais (Alvergnas et al., 2019; Palmer et al., 2012; Silva et al., 2006; Souza et al., 2006).

O modelo conceitual (Figura 5) enfatiza que, por natureza, o processo de tratamento das podopatias na assistência veterinária é de carácter multifatorial e coordenado envolvendo assistência à saúde dos animais por meio de clínica das podopatias (B1) e clínica da dor (B2). De fato, observa-se no diagrama que desta integração emerge o ciclo B3 que indica que as dinâmicas da dor e do sofrimento dependem tanto dos procedimentos de tratamento das podopatias quanto dos procedimentos de controle da dor. As podopatias e a dor causam sofrimento nos animais (Shearer et al., 2013; Silva et al., 2006). O sofrimento dos animais acometidos pelas podopatias, por hipótese, quando reconhecidos e percebidos resultam em ações que reforçam o Tratamento gerando os ciclos de controle (B4, B5 e B6) e melhorando o estado de bem-estar animal.
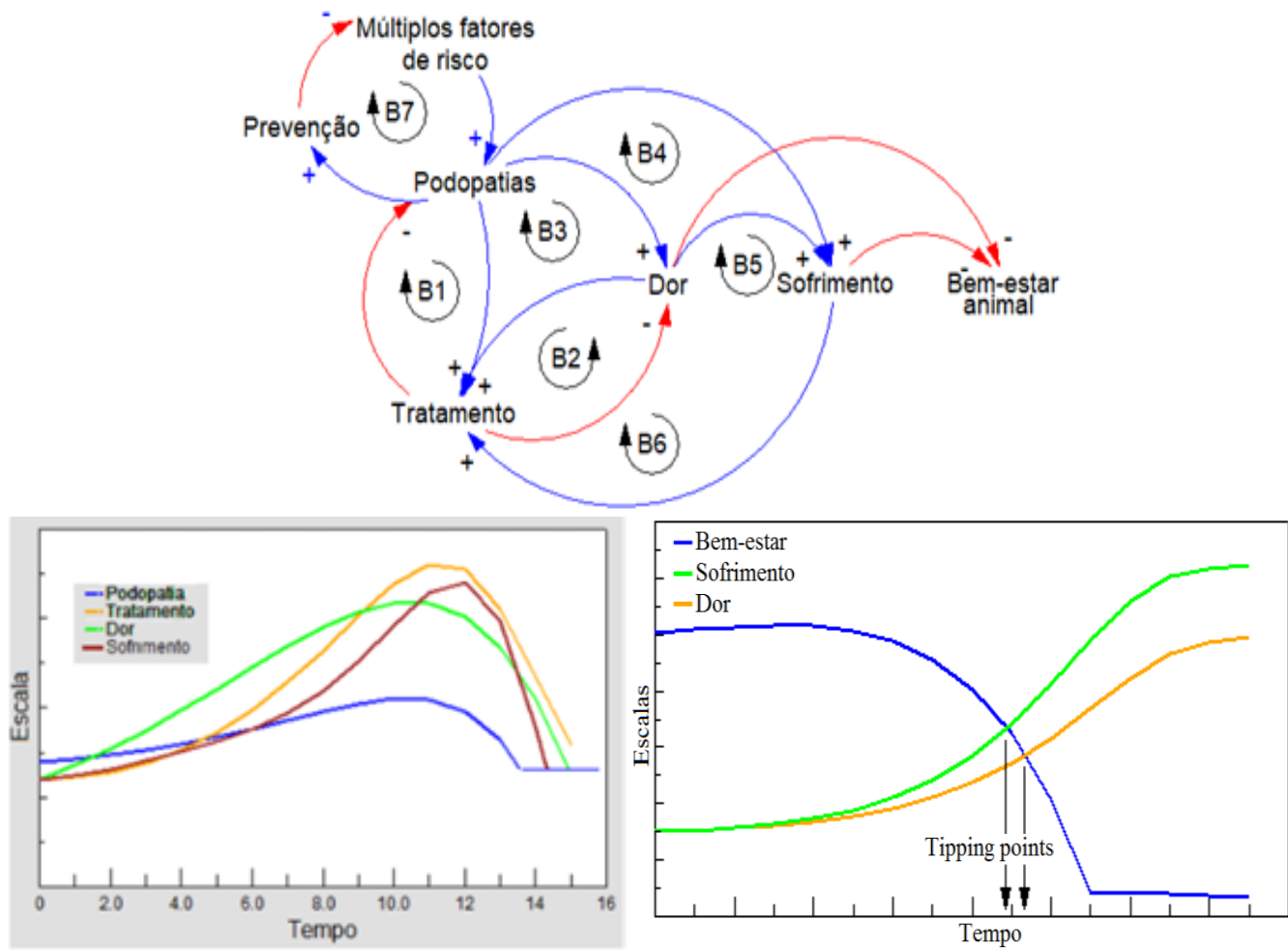

Figura 5. Diagrama de ciclos causais explicitando a hipótese dinâmica da formação de ciclos de balanço pelas influências com polaridades negativas das variáveis tratamento e prevenção. Os ciclos de retroalimentação de balanço (B1, B2, B3) indicam como a Dor e a ocorrência de Podopatias são controladas ao longo do tempo pelo Tratamento. Os ciclos B4, B5 e B6 estendem os efeitos do Tratamento para o controle do Sofrimento. O ciclo de balanço B7 indica a ação de controle (-) da prevenção sobre os fatores de risco. Quando a força das interações entre os ciclos de balanço predomina, o estado do sistema tende a um patamar de equilíbrio apresentado nos gráficos BOT de referência. A curva de intensificação de Tratamento (controle) está associada à diminuição das causas da ocorrência de podopatias e ao controle da dor e sofrimento. $\mathrm{O}$ aumento de práticas de Prevenção produz o mesmo padrão de efeito dinâmico de diminuição dos fatores de risco. 
No gráfico BOT (Figura 5, esquerda), as trajetórias das curvas projetam a influência da demora no efeito do tratamento clínico das podopatias sobre a diminuição da ocorrência de dor e sofrimento que tende para um patamar estável significando a recuperação clínica, enquanto que a dor e sofrimento continuam por algum tempo em níveis elevados, mesmo com o declínio da ocorrência das podopatias. No início do processo de ocorrência das podopatias, por hipótese, há a predominância dos ciclos de balanço B2 e B3 sobre o estado de saúde do sistema. O ciclo de balanço B1 exerce a função de controle sobre a ocorrência de podopatias (Ollhoff \& Ortolani, 2001; Neveux et al, 2006; Souza et al., 2006; Olmos et al., 2009; Amaral \& Trevisan, 2017). A influência da dor sobre o sofrimento gera outros dois ciclos de balanço, o B5 com caminho dor-sofrimento-tratamento-dor e o B6 com caminho dorsofrimento-tratamento-podopatias-dor. As trajetórias das podopatias, dor e sofrimento tendem a um valor mínimo devido ao processo de intensificação do controle.

No gráfico BOT (Figura 5, direita), o bem-estar dos animais diminui gradativamente com o aumento da dor e sofrimento devido a um círculo vicioso, não desejado, onde ocorre aumento da influência de fatores de risco que causam as podopatias que, na ausência de tratamento e prevenção adequados, terão seus quadros agravados causando, cada vez mais ao longo do tempo, o aumento da dor e sofrimento. $\mathrm{O}$ sofrimento está em um nível superior ao da dor por ser influenciado simultaneamente pela dor e pelas podopatias. A progressão da dor e sofrimento causados por podopatias é um processo dinâmico que ocorre de forma não linear a partir de um estado normal por influência de fatores de risco que provocam mudanças gradativas no estado do bem-estar animal. As curvas BOT (direita) indicam que, eventualmente, as podopatias resultam em uma transição drástica do bem-estar animal para um estado crítico, ou pontos de inflexão (tipping point), a partir do qual não é possível a reversão do bem-estar ao estado normal. Assim, ações de controle das podopatias são efetivas quando ocorrem antes do tempo do tipping point.

\section{Dinâmica dos processos veterinários e prevenção das podopatias}

O tratamento da dor em bovinos é extremamente importante dentro dos sistemas de produção, por isso a dor deve ser reconhecida, avaliada e tratada nos casos de desconforto e sofrimento animal para promover o bem-estar e melhorias na produção (Nunes et al., 2021).

No modelo conceitual (Figura 6) destacam-se as influências com polaridade positiva dos procedimentos veterinários de diagnóstico e controle que direcionam a dinâmica do sistema ao equilíbrio por meio de tratamento e prevenção das podopatias e do controle da dor. No gráfico BOT (Figura 6) observa-se que as podopatias e a dor associada crescem ao longo do tempo e a demora dos efeitos das influências do tratamento e da prevenção na diminuição das podopatias. Este processo é dependente da demora no diagnóstico das podopatias.
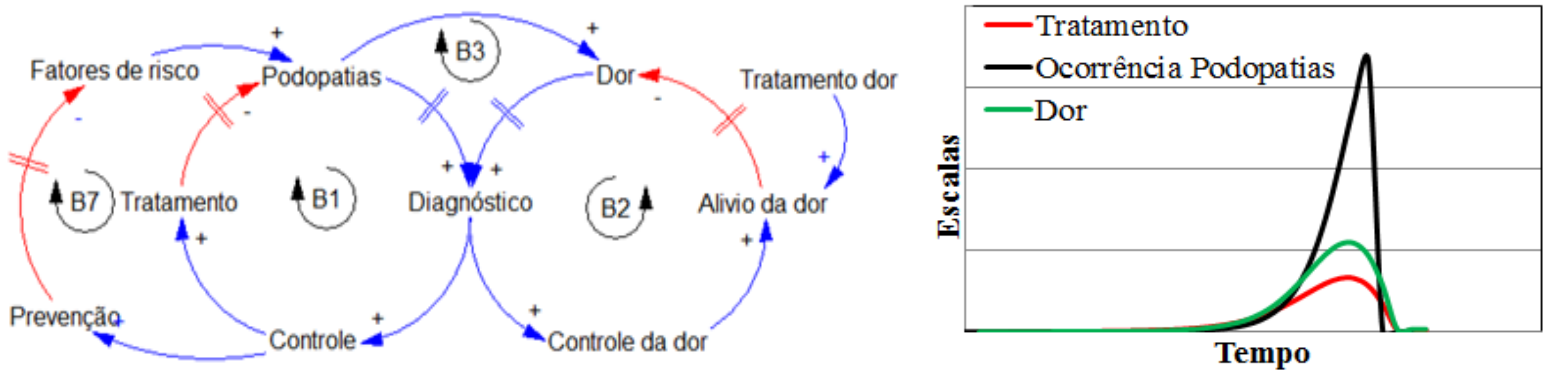

Figura 6. Diagrama de ciclos causais dos processos dinâmicos da atuação veterinária no tratamento e prevenção das podopatias e controle da dor que formam os ciclos de retroalimentação de balanço (B1, B2, B3 e B7) que equilibram os estados das podopatias e da dor ao longo do tempo. As setas com traços paralelos indicam demoras nas influências.

\section{Dinâmica das relações complexas econômicas no tratamento e prevenção das podopatias e controle da dor}

O conflito entre recursos financeiros escassos e a necessidade de investimento para assegurar a qualidade de vida dos animais afeta diretamente as atitudes em relação ao bem-estar dos animais de produção no Brasil. Ao entrar no mundo da economia, o bem-estar animal passa a ser parte integrante dos cálculos do valor econômico dos produtos de origem animal. As relações custo-benefício têm grande impacto nos países onde o bem-estar é uma demanda importante (Molento, 2005). Os possíveis desafios entre os sistemas produtivos e as necessidades dos animais podem ser minimizados pelos benefícios 
econômicos advindos da melhoria no bem-estar animal, com redução da taxa de mortalidade e morbidade, melhoria da saúde e menos custos com medicamentos (Braga et al., 2018).

Bruijnis et al. (2010) relataram em trabalho com modelagem, que as podopatias são importantes problemas de saúde no rebanho leiteiro, de custos de tratamento devido a perdas de produção de leite, de descarte de animal, do aumento do intervalo de parto, do trabalho do manejo do tratamento, dos custos do veterinário e leite descartado. Esta descoberta implica que os agricultores podem subestimar os benefícios de agir mais cedo e de forma mais completa e sistêmica. Pois, conforme os autores, um distúrbio clínico de uma afecção podal, em média, custa 5,27 vezes mais do que um distúrbio subclínico desta afecção. Assim, emerge da construção do modelo conceitual (Figura 7) que as decisões econômicas não estão desconectadas das decisões médico-veterinárias, quando se busca melhoria no bem-estar animal, em particular por meio de cuidados das podopatias na bovinocultura leiteira.

As podopatias são causas importantes do descarte de animais no rebanho de bovinos leiteiros por comprometerem a eficiência na reprodução e a diminuição na produção de leite (Romani et al., 2004; Silva et al., 2015; Souza et al., 2006). Estas relações estão representadas no submodelo rebanho por influências de polaridades positivas indicando que quanto maior a ocorrência de podopatias maior será a perda de animais. No modelo, a variável Descarte de animais influencia oito ciclos causais, suas relações diretas estão representadas nos ciclos de reforço R7 e R10 e no ciclo de balanço B12 (Figura 7). Os ciclos de reforço R7, R8 e R9 indicam as influências da ocorrência das podopatias sobre a estrutura do rebanho de bovinos leiteiros por meio de Descarte de animais e Mortes causando diminuição na quantidade de animais e, consequentemente, na diminuição de vacas em lactação. Quatro ciclos de Balanço (B1, B9, B10 e B11) centralizados pela variável Tratamento, a qual é influenciada com polaridade positiva pela variável Decisão por tratamento, atuam no controle dos efeitos das Podopatias sobre a estrutura do rebanho. Os ciclos B10 e B11 representam as decisões econômicas com base nas relações custo-benefício que têm predominado na bovinocultura leiteira em busca de patamares elevados de lucro ao longo do tempo.

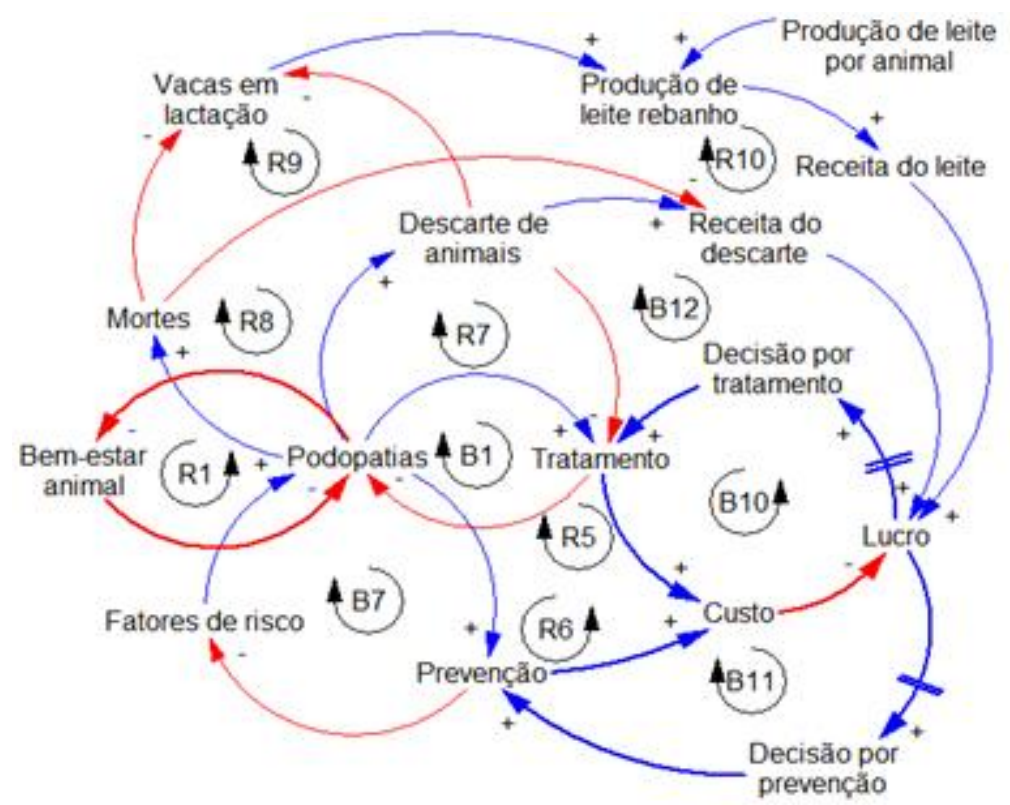

Figura 7. Diagrama de ciclos causais das relações complexas econômicas no tratamento e prevenção das podopatias e controle da dor. Esse sistema econômico é governado pelas interações entre ciclos de retroalimentação de balanço e reforço, com demoras significativas nos efeitos das influências que causam oscilações no estado do sistema de produção, em particular na ocorrência das podopatias.

A decisão do produtor na escolha das vacas a serem descartadas e quais serão mantidas no rebanho de reprodução afeta o desempenho futuro do rebanho e a lucratividade. A decisão por tratamento e/ou descarte de animais são influenciadas por critérios econômicos, mas é balizada por diagnóstico veterinário, quando se considera a integração dos modelos de processos veterinário e econômico. A complexidade das interações que ocorrem no modelo econômico entre os 15 ciclos de reforço e balanço, com presença de demoras nas influências, não permite estabelecer uma hipótese dinâmica de referência. 
Para tanto, é necessário construir um modelo computacional que possa ser validado com valores observados. Entretanto, as interações entre os ciclos de reforço (R5 e R6) e os ciclos de balanço (B1, B7, B10 e B11) podem ser entendidas usando a estrutura do arquétipo sistêmico chamado de princípio da atratividade (Senge et al., 2012) em que as decisões de liberar os ciclos de reforço de lucro (R5 e R6) limitam a ação dos ciclos de balanço de saúde (B1 e B7) que atuam no tratamento e prevenção para diminuir a ocorrência de podopatias e, consequentemente, na melhoria do bem-estar animal, ao longo do tempo.

\section{Dinâmica e implicações éticas, humanitárias e legais para o bem-estar animal}

O modelo conceitual considerou os valores técnicos, legais, éticos e humanitários que regem a bovinocultura leiteira para que a ocorrência das podopatias não ficassem estritamente sob controle de valores econômicos (Figura 8). O ciclo de retroalimentação negativa indica que a avaliação das podopatias, da dor e do sofrimento animal pela perícia veterinária, em conformidade com a legislação de bem-estar e proteção dos animais, exerce influência importante e contribui para adoção de ações humanitárias na melhoria da qualidade de vida na bovinocultura leiteira. Em termos legais, quanto menor o bem-estar mais grave será a classificação da Culpa, que demandará mais ações legais e forenses e aumento nas penalizações cabíveis, conforme a legislação.

O bem-estar animal quando classificado como baixo ou muito baixo configura maus-tratos, que é inaceitável. Neste caso, poderá ter implicações de culpas por negligência, imperícia ou imprudência nas esferas legais e forenses. Esta lógica dinâmica conduz o bem-estar dos animais para um patamar elevado no sistema de bovinocultura leiteira, pois o aumento de denúncias de maus-tratos e, consequentemente de penalizações, demanda por perícias veterinárias que aumentarão a conscientização da necessidade de adoção de boas práticas de manejo e criação dos animais, prevalecendo à ética, manejo humanitário e a melhoria do bem-estar dos animais, necessários também para atender as exigências do comércio nacional e internacional de produção animal e seus produtos.

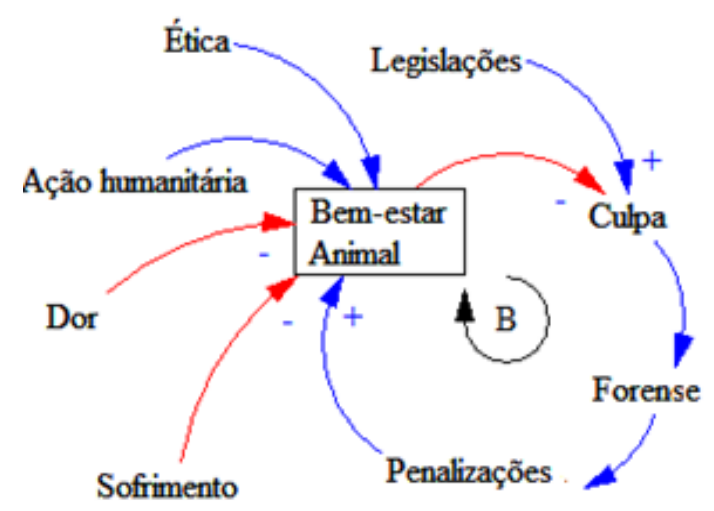

Figura 8. Diagrama de ciclos causais de processo legal e forense que influencia na melhoria do bem-estar animal. O aumento da dor e sofrimento em animais acometidos por podopatias diminui o estado de bem-estar desejado pelas pressões éticas, humanitárias e legais.

A perícia veterinária auxilia as autoridades judiciais na identificação das culpas por negligência, imperícia e imprudência, nos casos de maus-tratos, crueldade e abuso ou nos crimes contra a dignidade animal. Uma vez reconhecida a conduta culposa, seja por negligência, imperícia ou imprudência o infrator responderá na esfera administrativa ou até mesmo no âmbito jurídico (Slowinski et al., 2016). A ética sugere que o papel fundamental para o médico veterinário na sociedade está relacionado direta ou indiretamente às ações de controle da dor e do sofrimento no uso de animais, fazendo valer o bemestar animal (Amaral \& Trevisan, 2017). Ampliando a identificação de culpas aumentam-se as práticas forenses para esclarecê-las e a consequente penalização, quando cabível. Este processo contribui para elevar o padrão do bem-estar dos animais na bovinocultura leiteira, ao longo do tempo.

O grau de bem-estar dos animais depende do tipo e intensidade dos desafios enfrentados por eles. Para identificar esses desafios é preciso conhecer as necessidades dos animais, sendo importante uma visão do sistema produtivo (Carvalhal \& Costa, 2018). No âmbito científico, o bem-estar animal é 
constituído de uma escala que varia de grau muito alto a muito baixo e nesta classificação de bem-estar os graus baixo e muito baixo estão associados a uma qualidade de vida ruim. Quando se tem um grau de bem-estar baixo ou muito baixo, sem que o responsável pelos animais apresente ações que demonstram tentativas de evitar ou, pelo menos, de minimizar as dificuldades enfrentadas pelo animal, a situação poderá ser considerada inaceitável, sendo caracterizado como maus-tratos (Hammerschmidt \& Molento, 2017). No Brasil, os casos de maus-tratos aos animais estão sujeitos às sanções penais e administrativas, através no Art. 32, Cap. V, da Lei no 9.605/98, de Crimes Ambientais (BRASIL, 1998).

O grau de bem-estar regular é considerado aceitável se medidas corretivas forem asseguradas, enquanto que o grau alto e muito alto são desejáveis para o bem-estar animal. Esta terminologia pode ser utilizada nas decisões jurídicas relacionadas aos maus-tratos, facilitando os trabalhos no campo nas investigações (Hammerschmidt \& Molento, 2014). Pode-se também usar os indicadores de saúde que têm como princípio a identificação de dor, doenças ou ferimentos por meio do exame clínico do animal e das informações fornecidas pelo proprietário (Welfare Quality, 2009). Portanto, para reconhecer cientificamente o grau de bem-estar animal é importante a elaboração de técnica dinâmica de diagnóstico dos animais e do rebanho.

\section{Dinâmica multifatorial das podopatias, dor, sofrimento e bem-estar de bovinos leiteiros}

A integração dos ciclos de retroalimentação causal no modelo conceitual geral reforça a necessidade de conectar as decisões de diferentes níveis hierárquicos, desde o animal, passando pelo sistema de produção até a sociedade. Todos os ciclos de retroalimentação estão interconectados e o estudo da dinâmica multifatorial das podopatias e do bem-estar de bovinos leiteiros requer a construção de modelo computacional com base na estrutura destes modelos conceituais.

\section{Conclusão}

O modelo conceitual de Dinâmica de Sistemas enfatiza que, por natureza, o tratamento das podopatias na saúde animal é de carácter multifatorial e coordenado, envolvendo clínica médica, clínica da dor, medidas de prevenção e controle dos fatores de riscos. O modelo conceitual sugere fortemente que a metodologia de Dinâmica de Sistema pode ser usada como um método para facilitar a integração da saúde animal nas políticas e nos processos de tomada de decisão em setores não diretamente relacionados à saúde animal.

As demoras (delays) na transição entre os estados das podopatias dos animais nos rebanhos e seus efeitos sobre a dor, sofrimento e bem-estar, ao longo do tempo, estão sujeitos às retroalimentações entre os componentes do sistema de criação, manejo, ambiente e assistência médica veterinária e os bovinos leiteiros.

\section{Referências bibliográficas}

Alvergnas, M., Strabel, T., Rzewuska, K., \& Sell-Kubiak, E. (2019). Claw disorders in dairy cattle: Effects on production, welfare and farm economics with possible prevention methods. Livestock Science, 222, 54-64. https://doi.org/10.1016/j.livsci.2019.02.011.

Amaral, J. B., \& Trevisan, G. (2017). Aspectos da dor e sofrimento no bem-estar de bovinos leiteiros $\begin{array}{llll}\text { acometidos por } \quad \text { podopatias. } & \text { PUBVET, 11(11), }\end{array}$ https://doi.org/10.22256/pubvet.v11n11.1074-1084.

Amaral, J. B., Trevisan, G., Tremori, T. M., \& Guerra, S. T. (2018). Fundamentos e aplicações da medicina veterinária forense no bem-estar de bovinos leiteiros: Revisão. PUBVET, 12(2), 1-13. https://doi.org/10.22256/pubvet.v12n2a37.1-13.

Ataíde Júnior, V. (2018). Introdução ao direito animal brasileiro. Revista Brasileira de Direito Animal, 13(3), 48-76. https://doi.org/10.9771/rbda.v13i3.28768.

Berry, S. L., Read, D. H., Famula, T. R., Mongini, A., \& Döpfer, D. (2012). Long-term observations on the dynamics of bovine digital dermatitis lesions on a California dairy after topical treatment with lincomycin HCl. The Veterinary Journal, 193(3), 654-658. https://doi.org/0.1016/j.tvj1.2012.06.048.

Bicalho, R. C., Machado, V. S., \& Caixeta, L. S. (2009). Lameness in dairy cattle: A debilitating disease 
or a disease of debilitated cattle? A cross-sectional study of lameness prevalence and thickness of the digital cushion. Journal of Dairy Science, 92(7), 3175-3184. https://doi.org/10.3168/jds.2008-1827.

Bond B., G., Almeida, R., Ostrensky, A., \& Molento, C. F. M. (2012). Métodos e pontos críticos de bem-estar de bovinos leiteiros. Ciência Rural, 42, 1286-1293. https://doi.org/https://doi.org/10.1590/S0103-84782012005000044.

Braga, J. S., Macitelli, F., Lima, V. A., \& Diesel, T. (2018). O modelo dos “Cinco Domínios" do bemestar animal aplicado em sistemas intensivos de produção de bovinos, suínos e aves. Revista Brasileira de Zoociências, 19(2), 204-226.

BRASIL (2016). Conselho Federal de Medicina Veterinária - CFMV, Resolução nº 1138, de 16 de dezembro de 2016. Aprova o Código de Ética do Médico Veterinário.

BRASIL (2018). Conselho Federal de Medicina Veterinária - CFMV, Resolução no 1236, de 26 de outubro de 2018. Define e caracteriza crueldade, abuso e maus-tratos contra animais vertebrados. Dispõe sobre conduta de médicos veterinários e zootecnistas e dá outras providências.

Broom, D. M. (1986). Indicators of poor welfare. British Veterinary Journal, 142(6), 524-526.

Bruijnis, M. R. N., Beerda, B., Hogeveen, H., \& Stassen, E. N. (2012). Assessing the welfare impact of foot disorders in dairy cattle by a modeling approach. Animal, 6(6), 962-970. https://doi.org/10.1017/S1751731111002606.

Canozzi, M. E. A., Borges, J. A. R., \& Barcellos, J. O. J. (2020). Attitudes of cattle veterinarians and animal scientists to pain and painful procedures in Brazil. Preventive Veterinary Medicine, 177, 104909. https://doi.org/10.1016/j.prevetmed.2020.104909.

Capion, N., Thamsborg, S. M., \& Enevoldsen, C. (2008). Prevalence of foot lesions in Danish Holstein cows. Veterinary Record, 163(3), 80-86. https://doi.org/10.1136/vr.163.3.80.

Carvalhal, M. V. L., \& Costa, F. O. (2018). Principais aspectos sobre bem-estar de touros mantidos em centrais de coleta de sêmen. Revista Brasileira de Zoociências, 19(2), 249-264. https://doi.org/10.34019/2596-3325.2018.v19.24737.

Costa, F. O., Valente, T. S., de Toledo, L. M., Ambrósio, L. A., Campo, M., \& Paranhos da Costa, M. J. R. (2021). A conceptual model of the human-animal relationships dynamics during newborn handling on cow-calf operation farms. Livestock Science, 246, 104462. https://doi.org/10.1016/j.livsci.2021.104462.

Dawkins, M. S. (2017). Animal welfare and efficient farming: is conflict inevitable? Animal Production Science, 57(2), 201-208.

de Vries, M., Bokkers, E. A. M., van Schaik, G., Botreau, R., Engel, B., Dijkstra, T., \& de Boer, I. J. M. (2013). Evaluating results of the Welfare Quality multi-criteria evaluation model for classification of dairy cattle welfare at the herd level. Journal of Dairy Science, 96(10), 6264-6273. https://doi.org/http://dx.doi.org/10.3168/jds.2012-6129

Feitosa, F. L. F. (2014). Semiologia veterinária: A arte do diagnóstico. Grupo Gen-Editora Roca Ltda.

Flecknell, P. A. (2008). Analgesia from a veterinary perspective. British Journal of Anaesthesia, 101(1), 121-124. https://doi.org/https://doi.org/10.1093/bja/aen087.

Forrester, J. W. (1971). Counterintuitive behavior of social systems. Theory and Decision, 2(2), 109140. https://doi.org/10.1007/BF00148991.

Gaynor, J. S., \& Muir, W. W. (2009). Manual de controle da dor em medicina veterinária (Vol. 1). MedVet.

Gleerup, K. B., Andersen, P. H., Munksgaard, L., \& Forkman, B. (2015). Pain evaluation in dairy cattle. Applied Animal Behaviour Science, 171, 25-32.

Gleerup, K. B., Forkman, B., Otten, N. D., Munksgaard, L., \& Andersen, P. H. (2017). Identifying pain behaviors in dairy cattle. WCDS Advances in Dairy Technology, 29, 231-239.

Groves, J. T. (2018). Becoming indispensable by using" Systems Thinking" to tackle challenging and complex problems in practice. American Association of Bovine Practitioners Proceedings of the Annual Conference, 111-112. https://doi.org/10.21423/aabppro20183118.

Hammerschimidt, J., \& Molento, C. F. M. (2017). Perícia em bem-estar animal nos crimes de maus- 
tratos contra animais. In R. A. Tostes, S. T. J. Reis, \& V. V Castilho (Eds.), Tratado de medicina veterinária legal. Medvep.

Hammerschmidt, J., \& Molento, C. F. M. (2014). Protocolos de perícias em bem-estar animal como subsídio para decisões judiciais em casos de maus-tratos contra animais. Congresso Brasileiro de Bioética e Bem-Estar Animal, 423-426, Curitiba, Paraná, Brasil.

Hemsworth, P. H., Barnett, J. L., Beveridge, L., \& Matthews, L. R. (1995). The welfare of extensively managed dairy cattle: A review. Applied Animal Behaviour Science, 42(3), 161-182. https://doi.org/http://dx.doi.org/10.1016/0168-1591(94)00538-P.

IASP - International Association for Study of Pain (2020). https://dororofacial.com.br/definicao-de-doriasp-2020/ [Acesso 14 de Jun de 2021].

INFOPÉDIA - Dicionários Porto Editora 2021. https://www.infopedia.pt/dicionarios/termosmedicos/tratamento. [Acesso 14 de Jun de 2021].

Katzenberger, K., Rauch, E., Erhard, M., Reese, S., \& Gauly, M. (2020). Evaluating the need for an animal welfare assurance programme in South Tyrolean dairy farming. Italian Journal of Animal Science, 19(1), 1146-1156. https://doi.org/10.1080/1828051X.2020.1823897.

Lorena, S. E. R. S., Luna, S. P. L., Lascelles, B. D. X., \& Corrente, J. E. (2013). Attitude of Brazilian veterinarians in the recognition and treatment of pain in horses and cattle. Veterinary Anaesthesia and Analgesia, 40(4), 410-418. https://doi.org/10.1111/vaa.12025.

Luna, S. P. L. (2008). Dor, senciência e bem-estar em animais. Ciência Veterinária Nos Trópicos, 11(1), $17-21$.

Manson, F. J., \& Leaver, J. D. (1988). The influence of dietary protein intake and of hoof trimming on lameness in dairy cattle. Animal Science, 47(2), 191-199. https://doi.org/10.1017/S0003356100003263.

McLennan, K. M. (2018). Why pain is still a welfare issue for farm animals, and how facial expression could be the answer. Agriculture, 8(8), 127. https://doi.org/10.3390/agriculture8080127.

Molento, C. F. M. (2005). Bem-estar e produção animal: Aspectos econômicos - Revisão. Archives of Veterinary Science, 10(1), 1-11.

Morton, D. B., \& Hau, J. (2002). Welfare assessment and humane endpoints. In J. Hau \& G. L. van Hooser (Eds.), Handbook of laboratory animal science: essential principles and practices (Vol. 1, pp. 457-486). Seattle, CRC Press. https://doi.org/10.1201/b10416-19.

Neveux, S., Weary, D. M., Rushen, J., von Keyserlingk, M. A. G., \& Passillé, A. M. (2006). Hoof Discomfort Changes How Dairy Cattle Distribute Their Body Weight. Journal of Dairy Science, 89(7), 2503-2509. https://doi.org/http://dx.doi.org/10.3168/jds.S0022-0302(06)72325-6

Nicoletti, J. L. M. (2004). Manual de podologia bovina. Manole.

Nunes, M. H. V., Pacheco, A. D., \& Wagatsuma, J. T. (2021). Reconhecimento e avaliação da dor em bovinos: Revisão. PUBVET, 15(6), 1-12. https://doi.org/10.31533/pubvet.v15n06a831.1-12.

Ollhoff, R. D., \& Ortolani, E. L. (2001). Comparação do crescimento e do desgaste do casco em bovinos taurinos e zebuínos. Ciência Rural, 31, 67-71. https://doi.org/10.1590/s0103-84782001000100011.

Olmos, G., Boyle, L., Hanlon, A., Patton, J., Murphy, J. J., \& Mee, J. F. (2009). Hoof disorders, locomotion ability and lying times of cubicle-housed compared to pasture-based dairy cows. Livestock Science, 125(2-3), 199-207. https://doi.org/10.1016/j.livsci.2009.04.009.

Palmer, M. A., Law, R., \& O'Connell, N. E. (2012). Relationships between lameness and feeding behaviour in cubicle-housed Holstein-Friesian dairy cows. Applied Animal Behaviour Science, 140(3-4), 121-127. https://doi.org/10.1016/j.applanim.2012.06.005.

Payne, J. M. (1972). Production disease. Journal of the Royal Agricultural Society of England, 133, 6986.

Pinto, A. L. M., Vieira, F. V. R., Garcia, P. R., \& Silva, I. J. O. (2020). Manual of good practices for welfare: a proposal for dairy cattle on pasture in Brazil. Journal of Animal Behaviour and Biometeorology, 1(2), 44-51. https://doi.org/10.14269/2318-1265.v01n02a03.

Queiroz, M. L. V., Barbosa Filho, J. A. D., Albiero, D., Brasil, D. F., \& Melo, R. P. (2014). Percepção 
dos consumidores sobre o bem-estar dos animais de produção em Fortaleza, Ceará. Revista Ciência Agronômica, 45, 379-386. https://doi.org/10.17224/energagric.2019v34n4p201-510.

Ramanoon, S. Z., Sadiq, M. B., Shaik Mossadeq, W. M., Mansor, R., \& Syed-Hussain, S. S. (2018). The impact of lameness on dairy cattle welfare: Growing need for objective methods of detecting lame cows and assessment of associated pain. Animal Welfare. https://doi.org/10.5772/intechopen.75917.

Richardson, G. P. (1995). Loop polarity, loop dominance, and the concept of dominant polarity (1984). System Dynamics Review, 11(1), 67-88. https://doi.org/10.1002/sdr.4260110106.

Romani, A. F., Silva, L. A. F. ., \& Fioravanti, M. C. S. (2004). Ocorrência de lesões podais em fêmeas bovinas leiteiras no Estado de Goiás. ARS Veterinaria, 20(3), 322-392.

Senge, P. M. (2005). La quinta disciplina en la práctica. Ediciones Granica SA.

Shearer, J. K., Stock, M. L., Van Amstel, S. R., \& Coetzee, J. F. (2013). Assessment and management of pain associated with lameness in cattle. Veterinary Clinics: Food Animal Practice, 29(1), 135156. https://doi.org/10.1016/j.cvfa.2017.02.009.

Silva, F. F., Alves, C. G. T., \& Silva Júnior, F. F. (2016). Pododermatite solar circunscrita, úlcera de husterholz ou úlcera da sola. Ciência Veterinária Nos Trópicos, 9(2/3), 102-105.

Silva, L A F, Campos, S. B. S., Rabelo, R. E., Vulcani, V. A. S., Noronha Filho, A. D. F., \& Freitas, S. L. R. (2015). Análise comparativa da morfometria do casco de bovinos das raças Nelore, Curraleira e Pantaneira e de bubalinos e sua relação com a etiopatogenia das enfermidades digitais. Pesquisa Veterinária Brasileira, 35(4), 377-384.

Silva, L A F, Rezende, M. R., Romani, A. F., Fioravanti, M. C. S., Cunha, P. H. J., Borges, J. R. J., Macedo, S. P., Damasceno, A. D., Rabelo, R. E., \& Garcia, A. M. (2006). Pododermatite séptica em bovinos: evolução clínica da fase inicial. Brazilian Journal of Veterinary Research and Animal Science, 43(5), 674-680.

Slowinski, K., Tremori, T. M., Massad, M. R. R., Tasaka, A. C., \& Rocha, N. S. (2016). Responsabilidade ética e civil do médico-veterinário no ambiente hospitalar. Revista de Educação Continuada Em Medicina Veterinária e Zootecnia Do CRMV-SP, 14(2), 30-37.

Souza, R. C., Ferreira, P. M., Molina, L. R., Carvalho, A. U., \& Facury Filho, E. J. (2006). Perdas econômicas ocasionadas pelas enfermidades podais em vacas leiteiras confinadas em sistema free stall. Arquivo Brasileiro de Medicina Veterinária e Zootecnia, 58, 982-987. https://doi.org/10.1590/S0102-09352006000600002.

Sterman, J. (2000). Business dynamics. McGraw-Hill, Inc.

Ting, S. T. L., Earley, B., Hughes, J. M. L., \& Crowe, M. A. (2003). Effect of ketoprofen, lidocaine local anesthesia, and combined xylazine and lidocaine caudal epidural anesthesia during castration of beef cattle on stress responses, immunity, growth, and behavior. Journal of Animal Science, 81(5), $1281-1293$.

Toledo, L. M., Fernandes, T. B., Costa, M. J. R. P., \& Ambrósio, L. A. (2018). Modelling the dynamics of cow-calf dyadic behavior. International Journal of System Dynamics Applications, 7(4), 1-19. https://doi.org/10.36440/recmvz.v11i3.17373.

Tremori, T. M., \& Rocha, N. S. (2013). Exame do corpo de delito na Perícia Veterinária (ensaio). Revista de Educação Continuada Em Medicina Veterinária e Zootecnia Do CRMV-SP, 11(3), 30-35.

Van Dyke, R., Miele, A., \& Connor, M. (2021). An Investigation into the perceptions of veterinarians towards calf welfare in New Zealand. Animals, 11(2), 421. https://doi.org/10.3390/ani11020421.

von Keyserlingk, M. A. G., Rushen, J., Passillé, A. M., \& Weary, D. M. (2009). Invited review: The welfare of dairy cattle-Key concepts and the role of science. Journal of Dairy Science, 92(9), 41014111. https://doi.org/http://dx.doi.org/10.3168/jds.2009-2326

Voyer, J., \& Jordan, T. (2018). Veterinary telemedicine: A system dynamics case study. Systems, 6(1), 6. https://doi.org/10.3390/systems6010006.

Weary, D. M., \& Von Keyserlingk, M. A. G. (2017). Public concerns about dairy-cow welfare: how should the industry respond? Animal Production Science, 57(7), 1201-1209. https://doi.org/10.1071/an16680. 
Welfare Quality (2009), Welfare Quality assessment protocol for cattle. Welfare Quality Consortium, Lelystad, Netherlands.

Wilson, G., Bryan, J., Cranston, K., Kitzes, J., Nederbragt, L., \& Teal, T. K. (2017). Good enough practices in scientific computing. PLoS Computational Biology, 13(6), e1005510. https://doi.org/10.1371/journal.pone.0189878.

Yang, D. A., Laven, R. A., Müller, K. R., \& Gates, M. C. (2020). Modelling the transmission dynamics of bovine digital dermatitis in New Zealand pastoral dairy production systems. Veterinary Research, 5l(1), 1-10. https://doi.org/10.1186/s13567-020-00750-8.

Histórico do artigo:

Recebido: 3 de outubro de 2021

Aprovado: 14 de novembro de 2021

Disponível online: 30 de janeiro de 2022
Licenciamento: Este artigo é publicado na modalidade Acesso Aberto sob a licença Creative Commons Atribuição 4.0 (CC-BY 4.0), a qual permite uso irrestrito, distribuição, reprodução em qualquer meio, desde que o autor e a fonte sejam devidamente creditados. 